\title{
Correcting STEM distortions in atomically resolved elemental maps
}

Pavel Potapov ${ }^{1}$, Axel Lubk ${ }^{2}$, Martin Kamp ${ }^{3}$, Martin Stuebinger ${ }^{3}$, Ralph Claessen $^{3}$ and Michael Sing ${ }^{3}$

${ }^{1}$ IFW-Dresden, Dresden, Sachsen, Germany, ${ }^{2}$ IFW Dresden, Germany, United States, ${ }^{3}$ JuliusMaximilians-University, United States

During last decades, STEM EELS or EDS spectrum-imaging became a standard tool in materials science. STEM spectrum-imaging is capable to deliver elemental maps with atomic resolution and therefore to characterize ordered structures, coherent interfaces and 2D materials at the sub-nanometer level. In particular, the type of lattice termination at the interfaces is of interest as it affects crucially the electronic surface states [1]. However, spectrum-imaging techniques require (unless the multi-frame strategies are used) an order of magnitude slower scanning compared to classical STEM. This magnifies the image distortions related to the sample drift and environmental instabilities.

The distortions in atomically resolved spectrum-images can be revealed by comparing a complementary (recorded simultaneously with a spectrum-image) Annular Dark Field (ADF) image with an ideal crystal lattice contrast expected for a given material $[2,3]$. Then, spectrum-images can by corrected by using a warp operation, which uses bilinear interpolation.

In the present work, we develop further the approach $[2,3]$ and introduce a software tool for a posteriori processing of atomically resolved elemental maps. In contrast to [3], we avoid the interpolation operations in a spectrum-image as it might distort the statistical pattern of spectra and provoke processing issues in the curse of further profile fitting or PCA. Instead, we apply warping and interpolation to the extracted elemental maps and therefore facilitate greatly their interpretability. An overview ADF image acquired in fast scan mode before spectrum-imaging is utilized as a reference for evaluation of linear and non-linear distortions during slow scanning. Finally, the elemental maps are up-sampled to fit the resolution of the overview image, which improves their appearance while not increasing the size of the original spectrumimage.

Acknowledgement:

The authors appreciate support from ERC (grant 715620 under the Horizon 2020 program) and DFG project 431448015. 


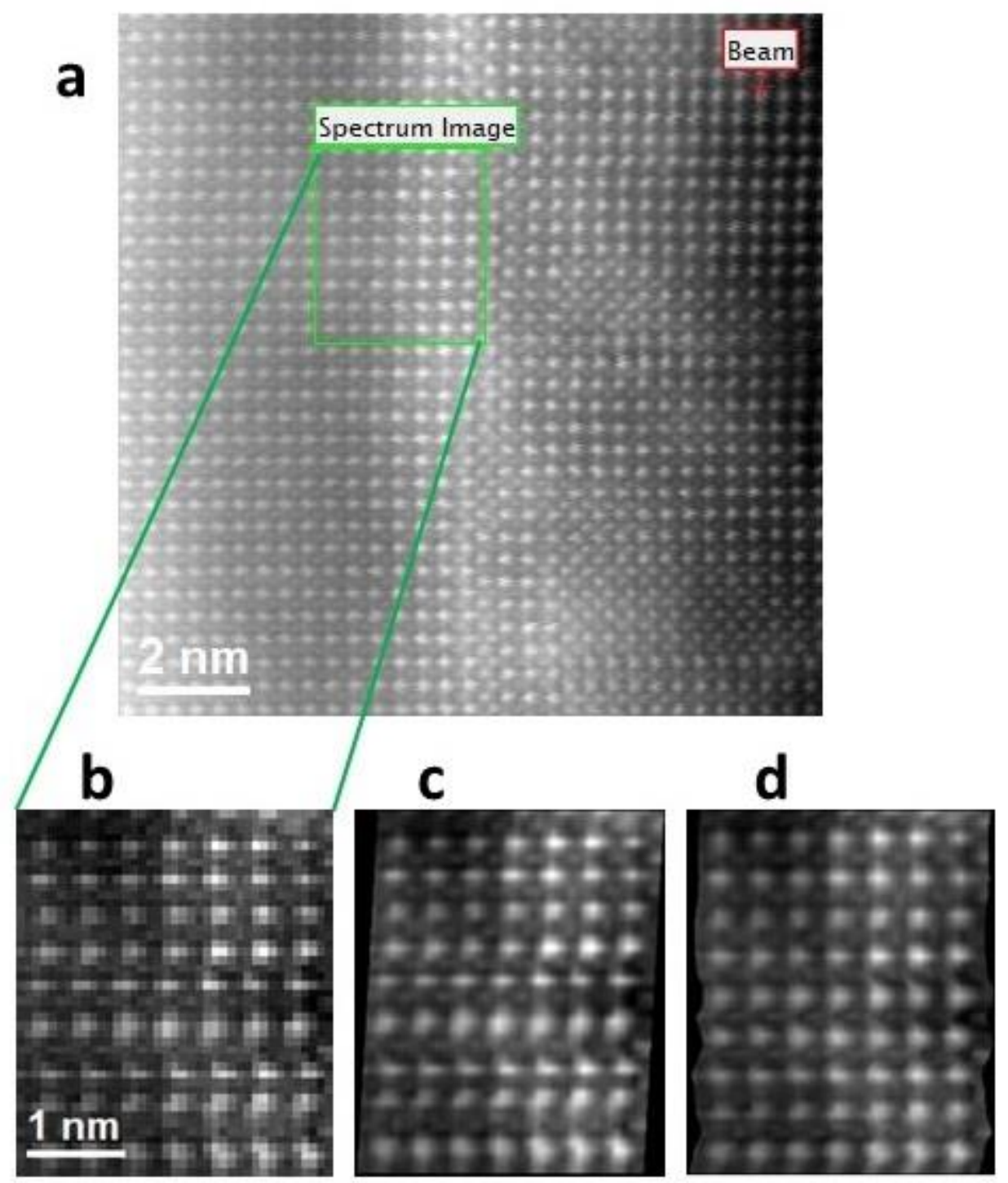

Figure 1. (a) Overview STEM ADF image of the SrTiO3 /LaFeO3 layered structure with the spectrumimaging area outlined by a green rectangular. (b) ADF image taken simultaneously with a spectrumimage. STEM distortions are clearly seen. (c) same image subjected to linear correction. (d) same image subjected to non-linear correction. 
a
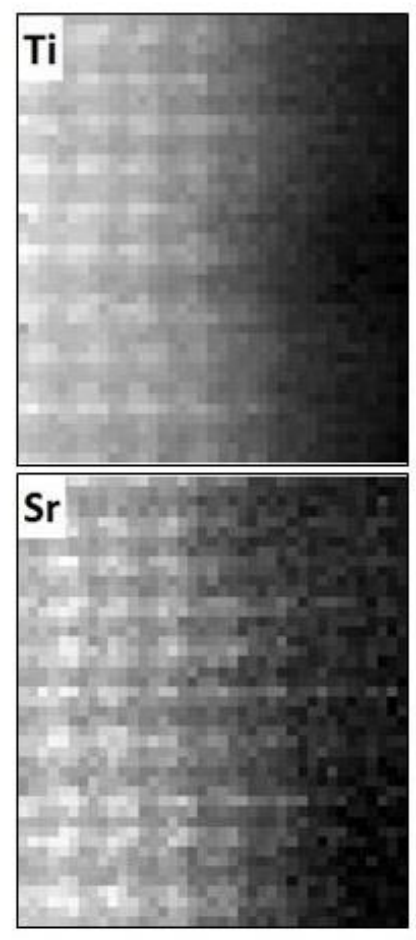

b

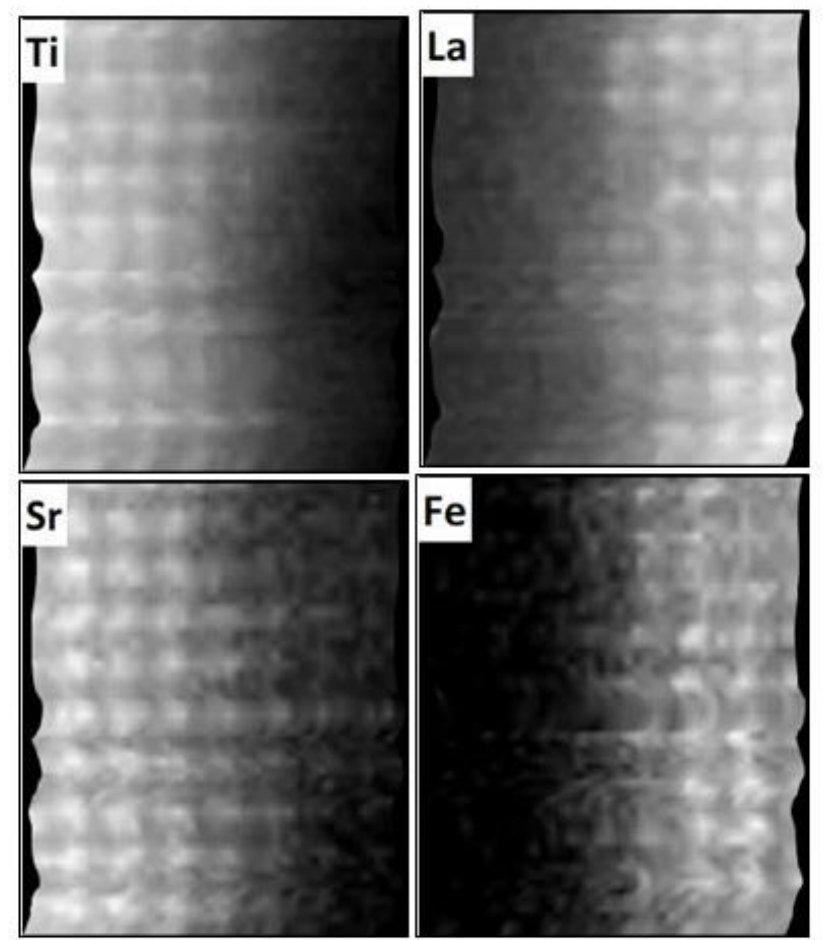

Figure 2. (a) Original (distorted) elemental maps extracted from the spectrum-image. (b) Elemental maps corrected with using the warping function evaluated from Fig.1d.

\section{References}

[1] P.Schuetz et al. Applied Physics Letters 116 (2020) 201601.

[2] Y. Wang et al. Microscopy and Microanalysis 23(S1) (2017) 408.

[3] Y. Wang et al. Microscopy 67 (2018) i114, https://doi.org/10.1093/jmicro/dfy002 